\title{
PERFLL INTRACOMUNITÁRIO DA DEFICIÊNCIA NUTRICIONAL: ESTUDO DE CRIANÇAS ABAIXO DE 5 ANOS NUMA COMUNIDADE DE BAIXA RENDA DO RIO DE JANEIRO (BRASIL)*
}

\author{
Michael Eduardo Reichenheim** \\ Trudy Harpham***
}

\begin{abstract}
REICHENHEIM, M.E. \& HARPHAM, T. Perfil intracomunitário da deficiência nutricional: estudo de crianças abaixo de 5 anos numa comunidade de baixa renda do Rio de Janeiro (Brasil). Rev. Saúde públ., S.Paulo, 24: 69-79, 1990.
\end{abstract}

RESUMO: Como parte de um estudo epidemiológico sobre a saúde de crianças abaixo de cinco anos realizado na favela da Rocinha, Rio de Janeiro, RJ (Brasil), avaliou-se o perfil nutricional de uma amostra representativa de 591 crianças. De acordo com o indicador peso-para-idade, $23,9 \%$ encontravam-se com desnutrição leve (grau I pela classificação de Gomez), e apenas 22,0\% evidenciaram desnutrição moderada (grau II). Esse achado mostrou-se compatível com aqueles onde se utilizaram os indicadores peso-paraaltura e altura-para-idade: (a) ausência de desnutriçăo aguda, com um perfil de peso-para-altura superposto ao de uma população padrão normal, e (b) deficiência de crescimento, com $7 \%$ e $15 \%$ de crianças excedendo os valores abaixo de, respectivamente, -2 e -1 desvios-padrão esperados numa população normal. Quanto à deficiência estatural, as seguintes variáveis mostraram-se associadas mesmo após controle pelo "status" econômico (indicado pelas condiçōes ambientais do domicilio): baixo peso-ao-nascer, número de irmãos igual ou acima de três, sexo masculino, história de nunca ter amamentado ao seio materno, e história de morte infantil prévia na família. Cada variável é discutida separadamente, bem como o perfil nutricional geral e a marcada estratificação social intracomunitária da dcficiência estatural.

DESCRITORES: Estado nutricional. Estatura. Peso corporal. Crescimento.

\section{INTRODUÇÃO}

A questão da desnutrição nas áreas urbanas não tem, até aqui, sido enfocada de forma esclarecedora. Alguns trabalhos têm mostrado que esta tende a ser menos severa do que a desnutrição encontrada nas zonas rurais $7,18,25,27,37,55,59$. Outros têm indicado que, à custa de uma transição demográfica rural-urbana, ocorrcu uma transferência do locus da desnutrição para os grandes centros 2,11, $20,34,39,50$, com o conseqüente acirramento dos diferenciais intra-urbanos $3,5,14,16,23$. Ainda outros autores mostram que nas zonas urbanas, como as das regiões sudeste e sul do Brasil, o perfil da desnutrição tem mudado, com a do tipo aguda tendendo a abrandar-se 4,33 e dando vazão à deficiência estatural crônica ${ }^{33}$.

E evidente que uma generalização não é possível no trato do assunto, nem tão pouco é produtiva. O perfil dos problemas nutricionais de cada zona urbana depende primordialmente de seu contexto, tornando irrelevantes as tentativas de encontrar um quadro definitivo. A perspectiva maior deve ser a de diagnosticar as condições vigentes num determinado local com vias à tomada de medidas adequadas.

Ainda que muitos estudos tenham chamado atenção para a determinação sócio-econômica intra-urbana da desnutrição infantil6, 15, 22, 24, 38, 44, 56, 62, uma questão ainda pouco explorada e de real importância no que tange a intervenções apropriadas concerne os diferenciais intracomunitários do perfil nutricional e de seus fatores determinantes. Esta lacuna toma vulto quando muitas das chamadas "comunidades de baixa-renda" já se assemelham em sua magnitude a grandes bairros ou até mesmo a cidades de porte médio.

Diante do acentuado aumento do número de favelas ocorrido no município do Rio de Janeiro na scgunda metade deste século ${ }^{8,42}$, questões referentes à distribuição da desnutrição em diferentes subpopulações de uma mesma comunidade urbana, e se cstas possíveis diferenças têm as mesmas nuances do que na população em geral, ainda não estão bem respondidas.

Este estudo faz parte de um inquérito de saúde de crianças abaixo de cinco ąnos realizado na favela da Rocinha, a maior do município do Rio de

* Realizado com auxílio financeiro do Consclho Nacional de Desenvolvimento Científico e Tecnológico - CNPq UNICEF-Brasil e CAFOD, Reino Unido.

* Departamento de Epidemiologia do Instituto de Medicina Social da Universidade do Estado do Rio de Janeiro Rua São Francisco Xavier, $524-7^{\circ}$ andar - 20550 - Rio de Janciro, RJ - Brasil. (Professor Visitante).

* * Evaluation and Planning Centre for I-Icalth Care, London School of Hygiene and Tropical Medicine, University of London - Keppel Street, London WCIE 1HT, England. 
Janeiro ${ }^{4 *}$. A favela ocupa uma área de aproximadamente meio quilômetro quadrado e fica localizada parcialmente em encostas montanhosas no coração da zona mais abastada da cidade (Zona Sul). Sua situação geográfica favoreceu o desenvolvimento de um amplo mercado de trabalho, ainda que em parte dentro do setor informal. No entanto, sua localização também estimulou uma crescente especulação imobiliária que acarretou uma visível estratificação espacial e econômica ${ }^{42}$. A indicação desse fenômeno pode ser obervada na Figura 1, que mostra o grau de concentração de renda domiciliar intracomunitário.

Com este pano de fundo, o presente artigo apresenta e discute as taxas de prevalência das deficiências ponderal e estatural da população abaixo de cinco anos. Diante do quadro geral identificado, são apresentados os fatores associados ao problema nutricional preponderante: 0 déficit de crescimento. As variáveis propostas para investigação foram escolhidas com base na vigente teoria sobre o assunto ${ }^{35}$ e são de três tipos: (a) variável econômica indicada pelas condiçōes ambientais do domicílio; (b) variáveis maternas - idade, grau de escolaridade, estado civil, paridade, falecimento prévio de filho/a e estado de migração; e (c) variáveis referentes à criança - idade, sexo, espaçamento gestacional, peso ao nascer e histórico de aleitamento.

\section{METODOLOGIA}

\section{Amostragem}

$O$ inquérito foi realizado em dois estágios entre julho e novembro de 1986 . Inicialmente uma malha de amostragem constituída de domicílios com mães de crianças abaixo de cinco anos foi estabelecida na base de censos em áreas da Rocinha, aleatoriamente escolhidas. A malha de amostragem do primeiro estágio consistiu de 30 áreas da Rocinha especificadas para o censo brasileiro de 1980. Mil e quarenta e oito mães com crianças abaixo de cinco anos foram encontradas nas 10 áreas levantadas. Subseqüentemente, foram aleatoriamente selecionadas 480 mảes para entrevistas domiciliares, correspondendo a 591 crianças pesadas e medidas. Isto equivale a uma taxa de resposta de $98,5 \%$. Detalhes do método de seleção e treinamento dos entrevistadores e sobre o desenho do estudo podem ser encontrados no trabalho sobre morbidade infantil e seus fatores de risco realizado na favela em questão42.

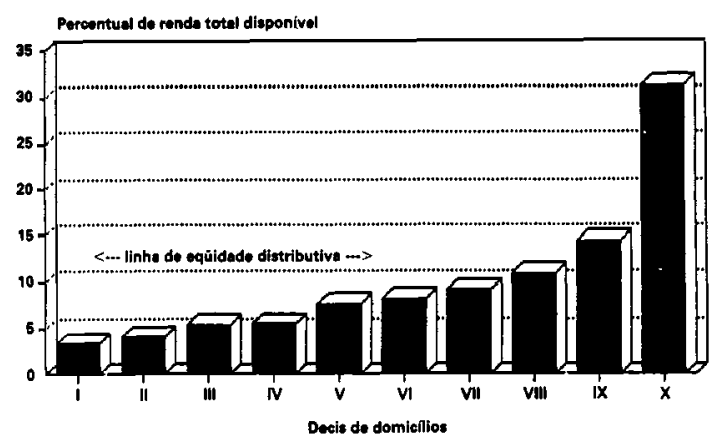

Fonto: Roichenheim, 1900

Fig. 1 - Distribuição de renda na favela da Rocinha, Rio de Janeiro, RJ, segundo os decis de distribuição de domicilios, 1986.

\section{Medidas Antropométricas}

As crianças foram pesadas com balanças suspensas CMS tipo-salt, modelo MP $25(25 \mathrm{~kg} \times 100 \mathrm{~g})$. A calibração foi feita todas as manhãs e antecedendo cada pesagem. O procedimento deu-se com a criança despida e foi realizado por entrevistadores especialmente treinados e auxiliados pelas respondentes (mães). As leituras foram arredondadas para o $0,1 \mathrm{~kg}$ mais próximo. A altura foi obtida através de antropômetros portáteis CMS com tara horizontal. Crianças com idade inferior a dois anos foram medidas em decúbito dorsal. Aquelas entre dois e dois e meio anos foram medidas tanto em decúbito dorsal quanto na posição ereta. As com idades acima de dois e meio anos foram medidas somente em pé. Em cinco ocasiozes a criança teve que ser trazida para o posto de saúde para ser medida pelo coordenador da pesquisa (MER) devido às dificuldades físicas ou psicológicas encontradas no domicilio.

Como a logística do estudo de campo impediu que dois entrevistadores operassem sistematicamente juntos, não foi possivel obter replicações independentes durante a coleta do material. $\mathrm{Na}$ medida em que tais problemas foram previstos, um esquema especial para avaliar a qualidade das mediçōes foi realizado ao final do período de treinamento dos entrevistadores. Para as replicações das medidas, utilizaram-se 30 crianças de creches locais. Cada entrevistador pesou e mediu de 8 a 12 crianças. Consideram-se vantagens deste procedimento os fatos de que: (a) as replicações puderam

* A populaçăo da Rocinha é uma questão bastante controversa. De acordo com o último censo, a população foi estimada em 32.966 habitantes [ IPLANRIO. Cadastramento de favelas no municipio do Rio de Janeiro. Estimatioa da populaçāo residente $e$ do número de domicilios particulares ocupados - Quadro II - dados obtidos a partir dos setores aglomerados do IBGE - 1980 e da estimativa do IPLANRIO - 1983 (mimeogr.)], ao passo que, localmente, especula-se que estes números cheguem aos 300.000 [Gomes LF. Médico de favela, o especialista em tudo. Jornal do Brasil, 24 de setembro de 1986, Rio de Janeiro]. De acordo com o presente inquérito, a populaçāo deve se encontrar em torno dos 80.000 . 
ser independentes, à medida que as comparaçōes eram feitas somente com as leituras do coordenador do projeto (MER) e assim realizadas isoladamente dos outros entrevistadores; e (b) permitiram uma avaliação anterior ao início do trabalho de campo, oferecendo uma maior segurança quanto à qualidade da atuação dos entrevistadores.

As replicagens de peso mostraram $76 \%$ de concordância completa, apenas quatro medidas ultrapassando 0,1 kg e uma média de diferença entre medidas de $0,027( \pm 0,103) \mathrm{kg}$. As replicagens de altura mostraram $30 \%$ de concordância completa, $77 \%$ de replicações com $\pm 1 \mathrm{~cm}$ do padrão (MER) e apenas quatro observaçठ̃es acima de $\pm 2 \mathrm{~cm}$ do padrão. A média de diferença (bruta) foi de $-0,048$ $( \pm 2,034) \mathrm{cm}$, i.e., $95 \%$ das medidas caindo entre $-2,1 \mathrm{~cm}$ e $2,0 \mathrm{~cm}$ da medição padrão. Ainda que as médias de diferenças tendam a zero, os dados apresentados no presente artigo devem ser analisados à luz desta variação extra incorrida por cada entrevistador.

Seguindo recomendações da Organização Mundial de Saúde ${ }^{60}$, utilizaram-se os parâmetros do National Center for Health Statistics (NCHSUSA) como referência para peso-para-altura e altura-para-idade. $O$ aval para o uso desta referência baseia-se no argumento de que diferenças étnicas são pequenas quando comparadas aos efeitos ambientais 19, 21, 29. Estudos brasileiros corroboram tais clamos, o que confiabiliza o seu uso como padrão para populações brasileiras ${ }^{28,61}$.

A preferência dada para peso-para-altura e altura-para-idade como indicadores do estado nutricional respalda-se no fato de que estes medem eventos diversos, enquanto o usual peso-paraidade é uma compilaçăo menos específica de ambos $^{61}$. Peso-para-altura mede o grau de perda tecidual recente e aguda (wasting). Altura-paraidade indica o grau de desaceleração ou estagnação do crescimento esquelético, representando um acumulado retardo de crescimento (stunting). Ambos os indicadores compreendem mais de $95 \%$ da variação de peso-para-idade ${ }^{61}$. A análise deste último indicador, pela da classificação de Go$\mathrm{mez}^{17}$, foi usada somente para efeito de comparação externa dos dados.

Seguindo a mesma orientação da Organização Mundial de Saúde ${ }^{60}$, as medições (peso e altura) foram relacionadas à população padrão do $\mathrm{NCHS}$ através de escores de desvio padrão - escores-z. $O$ cálculo individual de um escore-z para alturapara-idade, por exemplo, é dado por:

altura do indivíduo - valor mediano de altura para a idade da população de referência

$\mathrm{Z}=$

1 desvio padrão abaixo da mediana de altura para a idade da população de referência
A análise subseqüente pode se dar com a variável antropométrica na sua forma intervalar ou na ordinal, à conveniência da estratégia de análi-se escolhida. No presente estudo, optou-se pela segunda possibilidade.

\section{Medidas das outras variáveis}

As informaçôes sobre outras variáveis em estudo foram obtidas através de um questionário estruturado aplicado às mães. Os níveis de descrição e definições de cada variável podem ser encontrados na seção de resultados (texto e Tabela 3 ). Maiores detalhes podem ser encontrados em trabalho anterior ${ }^{42}$.

Para efeito de interpretação utilizou-se o escore ambiental como um indicador do nível econômico da familia (alguns detalhes sobre a variável "condições ambientais" são descritos na observação (b) da Tabela 3). Diante das muitas possibilidades de classificação do nível econômico ${ }^{26}$, a presente escolha baseou-se no seguinte argumento. Uma classificação por ocupação baseada na inserção setorial do arrimo de familia ignoraria o fato de que numa comunidade como a aqui estudada nāo há um diferencial de renda entre os setores formal e informal ${ }^{42}$. Isto levaria a um pequeno poder discriminativo do indicador, uma vez que o estado nutricional tem direta relação com o consumo alimentar e as condiçбes do meio em que vive a criança, e que em última análise sublinha uma questão eminentemente financeira. No entanto, sendo a renda familiar uma variável de alta flutuaçăo numa comunidade onde a estabilidade de emprego é muito baixa e onde o emprego no setor informal prepondera, procurou-se um indicador que fosse mais consistente ao longo do tempo. Isto permitiria, principalmente, uma melhor avaliação de relação entre o fator econômico-financeiro e o indicador altura-para-idade, cuja deficiência é cumulativa e contínua60,61. Martorell e col. ${ }^{30}$, estudando a relação entre as características antropométricas de crianças abaixo de sete anos e medidas de riqueza, também apontam para as condições ambientais como sendo o indicador mais informativo.

\section{Processamento e análise dos dados}

Para o processamento, checagem de consistência e recuperação dos dados, utilizou-se o "software" SIR/DBMS (Scientific Information Retrieval/Data Base Management System) ${ }^{51}$. Para a análise, dois "softwares" foram utilizados: SAS (Statistical Analysis System) ${ }^{49}$ para as análises de classificação cruzada e a sub-rotina do CDC (Centres for Disease Control - USA) ${ }^{9}$ para as análises antropométricas. Para a análise de precisão foram utilizados o método estatístico de Quiquadrado de Pearson para as tabulaçōes bivariadas e o método de Mantel-Haenszel para as análises estratificadas ${ }^{1}$. 


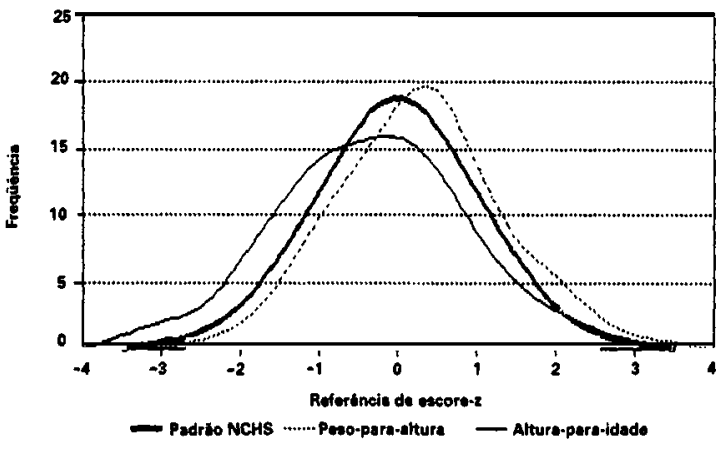

Fig. 2 - Perfil antropométrico da população abaixo de 5 anos da Rocinha, Rio de Janeiro, RJ, 1986.

\section{RESULTADOS}

\section{Perfil Nutricional das Crianças}

Peso-para-altura - A Figura 2 (linha pontilhada) mostra que a distribuição das crianças quanto ao peso-para-altura superpõe-se ao perfil esperado para uma população normal. Há mesmo uma pequena tendência para a obesidade. Sob outro ângulo, a Tabela 1 mostra o percentual acima do esperado de crianças que se encontravam além dos limites de +1DP e +2DP. Esta tendência foi mais pronunciada nos primeiros dois anos de idade com um excedente de, respectivamente, $9,3 \%$ e $8,8 \%$ além do limite de $+1 D P\left(\chi^{2}=14,34 ; p=0,073\right)$.

Altura-para-idade - O desvio para a esquerda em relação à altura-para-idade observado na Figura 2 (linha contínua) corresponde a $7 \%$ e 15\%, excedendo os limites inferiores de -2DP e-1DP esperados numa população de crianças normais (Tabela 1). Este perfil pode ser observado em todos os grupos etários, mas acentuou-se com idades crescentes. O excedente além do ponto de corte -1DP sobe de $9 \%$ no primeiro ano para $20 \%$ no quarto e quinto anos de vida $\left(\chi^{2}=15,733 ; p=0,046\right)$. O efeito cumulativo da deficiência estatural ao longo dos anos também tem sido descrito por outros autores ${ }^{54}$. 57,58 .

TABELA 2

Estado nutricional de acordo com a classificaçăo de Gomez

\begin{tabular}{cccc}
\hline \multicolumn{4}{c}{ Classificação de Gomez em Porcentagem } \\
\hline$>90 \%$ Med & $76-90 \%$ Med & $61-75 \%$ Med & $560 \%$ Med \\
\hline 74,1 & 23,9 & 2,0 & - \\
\hline$(N=590)$ & \multicolumn{4}{l}{}
\end{tabular}

Peso-para-idade - A Tabela 2 exibe o estado nutricional das crianças de acordo com a classificação de Gomez. Não se encontrou nenhuma criança com grau IIl de desnutrição. A maioria dos casos era de subnutrição de grau I. Dado as características do indicador peso-para-idade, esse perfil se coaduna com os achados descritos anteriormente, isto é, ausência de desnutrição aguda e presença de deficiência estatural.

Devido ao quadro observado, apenas serão mostrados a seguir os fatores associados com o déficit estatural (além do fator idade da criança já aventado). Este é aqui definido por uma variável dicotômica com ponto de corte de -1 desvio padrão.

\section{Fatores Associados ao Déficit de Crescimento}

Das variáveis propostas para estudo, apenas três referentes às características maternas não se mostraram discriminativas à analise bivariada: idade ( $<20$ anos v.s. $\geq 20$ anos), estado civil (morando com v.s. sem companheiro), e estado migratório (migrantes com $\leq 3$ anos, 4-10 anos e $>10$ anos na Rocinha e nascidas localmente). Os demais fatores associados a um nível de $\alpha=0.10$ de significância estatística são exibidos na Tabela 3. A condição econômica da família - aqui indicada pelas condições ambientais do domicnio - mostrouse um importante antecedente de déficit estatural,

TABELA 1

Percentual de crianças esperadas (numa população normal) e observadas cm categorias específicas de escores -z (DP), de acordo com o peso-para-altura c altura-para-idade.

\begin{tabular}{cccccc}
\hline $\begin{array}{c}\text { Ponto } \\
\text { de Corte }\end{array}$ & $\begin{array}{r}\text { Percentual } \\
\text { Esperado }\end{array}$ & \multicolumn{2}{c}{ Peso-para-Altura } & \multicolumn{2}{c}{ Altura-para-Idade } \\
\hline & & $\begin{array}{c}\text { Percentual } \\
\text { Observado }\end{array}$ & $\begin{array}{c}\text { Diferença } \\
\text { Percentual }\end{array}$ & $\begin{array}{c}\text { Percentual } \\
\text { Obscrvado }\end{array}$ & $\begin{array}{c}\text { Diferença } \\
\text { Percentual }\end{array}$ \\
\hline <-2 DP & 2,3 & 1,7 & $-0,6$ & 9,4 & 7,1 \\
$<-1$ DP & 15,9 & 12,4 & $-3,5$ & 31,1 & 15,2 \\
$>+1$ DP & 15,9 & 20,4 & 4,5 & 12,7 & $-3,2$ \\
$>+2$ DP & 2,3 & 5,2 & 2,9 & 3,7 & 1,4 \\
\hline
\end{tabular}


TABELA 3

Fatores associados ao déficit estatural de crianças da Roçinha, Rio de Janeiro, RJ, 1986

\begin{tabular}{|c|c|c|c|c|}
\hline $\begin{array}{l}\text { Fator associado ao } \\
\text { déficit estatural }\end{array}$ & $\begin{array}{l}\text { Casos por } \\
\text { estrato e total }\end{array}$ & $\begin{array}{c}\% \text { com } \\
\text { déficit estatural }\end{array}$ & $\begin{array}{c}\text { Razão de } \\
\text { produtos cruzados } \\
\text { (I.C. } 95 \% \text { (a)) }\end{array}$ & $\begin{array}{l}\text { Valor P de } \\
\text { significância }\end{array}$ \\
\hline \multicolumn{5}{|l|}{ Estrato econômico (b) } \\
\hline $\begin{array}{l}\text { Baixo } \\
\text { Médio } \\
\text { Alto }\end{array}$ & $\begin{array}{l}134 \\
290 \\
164 \\
588\end{array}$ & $\begin{array}{l}47 \\
29 \\
23\end{array}$ & $\begin{array}{l}3,1(1,8-5,5) \\
1,4(0,8-2,3) \\
1,0\end{array}$ & 0,000 \\
\hline \multicolumn{5}{|l|}{ Escolaridade materna (c) } \\
\hline $\begin{array}{l}\text { Não escolarizada } \\
\text { Escolarizada }\end{array}$ & $\begin{array}{l}348 \\
241 \\
589\end{array}$ & $\begin{array}{l}36 \\
25\end{array}$ & $\begin{array}{l}1,6(1,1-2,3) \\
1,0\end{array}$ & 0,011 \\
\hline \multicolumn{5}{|l|}{ Paridade } \\
\hline $\begin{array}{l}\text { Três ou mais crianças } \\
\text { Duas crianças } \\
\text { Uma criança }\end{array}$ & $\begin{array}{l}273 \\
170 \\
142 \\
585\end{array}$ & $\begin{array}{l}40 \\
25 \\
20\end{array}$ & $\begin{array}{l}2,6(1,5-4,5) \\
1,3(0,7-24) \\
1,0\end{array}$ & 0,000 \\
\hline \multicolumn{5}{|c|}{ Falecimento prévio de filho/a } \\
\hline $\begin{array}{l}\text { Positivo } \\
\text { Negativo }\end{array}$ & $\begin{array}{r}116 \\
469 \\
585\end{array}$ & $\begin{array}{l}41 \\
28\end{array}$ & $\begin{array}{l}1,8(1,2-27) \\
1,0\end{array}$ & 0,007 \\
\hline \multicolumn{5}{|l|}{ Sexo da criança } \\
\hline $\begin{array}{l}\text { Masculino } \\
\text { Feminino }\end{array}$ & $\begin{array}{l}298 \\
293 \\
591\end{array}$ & $\begin{array}{l}35 \\
27\end{array}$ & $\begin{array}{l}1,5(1,1-2,0) \\
1,0\end{array}$ & 0,037 \\
\hline \multicolumn{5}{|c|}{ Intervalo interpartal subseqüente (d) } \\
\hline $\begin{array}{l}\leq 24 \text { meses } \\
>24 \text { meses }\end{array}$ & $\begin{array}{r}85 \\
504 \\
589\end{array}$ & $\begin{array}{l}39 \\
30\end{array}$ & $\begin{array}{l}1,5(0,9-2,4) \\
1,0\end{array}$ & 0,090 \\
\hline \multicolumn{5}{|c|}{ Intervalo interpartal prévio (d) } \\
\hline $\begin{array}{l}\leq 24 \text { meses } \\
>24 \text { meses }\end{array}$ & $\begin{array}{l}126 \\
\frac{461}{587}\end{array}$ & $\begin{array}{l}40 \\
29\end{array}$ & $\begin{array}{l}1,6(1,1-2,5) \\
1,0\end{array}$ & 0,022 \\
\hline \multicolumn{5}{|l|}{ Peso-ao-nascer } \\
\hline $\begin{array}{l}>2,5 \mathrm{~kg} \\
2,5-3,8 \mathrm{~kg} \\
\geq 3,8 \mathrm{~kg}\end{array}$ & $\begin{array}{r}69 \\
423 \\
68 \\
560\end{array}$ & $\begin{array}{l}46 \\
28 \\
19\end{array}$ & $\begin{array}{l}3,7(1,6-8,3) \\
1,7(0,8-3,5) \\
1,0\end{array}$ & 0,001 \\
\hline \multicolumn{5}{|c|}{ Histórico de aleitamento natural (e) } \\
\hline $\begin{array}{l}\text { Nunca amamentou } \\
\text { Até } 4 \text { meses } \\
\text { Mais do que } 4 \text { meses }\end{array}$ & $\begin{array}{r}49 \\
320 \\
219 \\
588\end{array}$ & $\begin{array}{l}43 \\
32 \\
26\end{array}$ & $\begin{array}{l}2,1(1,1-4,1) \\
1,3(0,8-2,2) \\
1,0\end{array}$ & 0,066 \\
\hline
\end{tabular}

(a) Intervalo de confiança de $95 \%$.

(b) No presente estudo as condições ambientais são equacionadas ao padrão econômico intra-comunitário. As condições ambientais foram derivadas de um sistema de escores a partir das seguintes variáveis domiciliares: aglomeração, material de construção, material do piso, disponibilidade de eletricidade, tipo de saneamento interno, tipo de saneamento externo, origem da água e tipo de recolhimento do lixo. Os domicilios foram classificados como de estrato baixo com um escore ambiental entre 0 a 8 , de estrato médio com um escore de 9 a 13 , e de estrato alto com um escore de 14 a 17.

(c) A definição de não escolarizada inclue as mães que nunca freqüentaram escola e aquelas que não chegaram a concluir o primário (os quatro primeiros anos do atual $1^{\circ} \mathrm{grau}$ ).

(d) Com respeito ao intervalo prévio, crianças primogênitas ou únicas foram classificadas no grupo das com intervalo $>24$ meses. $O$ mesmo se deu $\mathrm{cm}$ relação ao intervalo subeqüente com as crianças caçulas, independente de suas idades.

(e) As crianças com menos de 4 meses e que ainda estavam amamentando ao peito à época da pesquisa foram classificadas no grupo das que amamentaram mais do que 4 meses. Isto lhes dá uma atribuição de forma ideal de aleitamento, precisamente o que esta categoria procurou captar. 


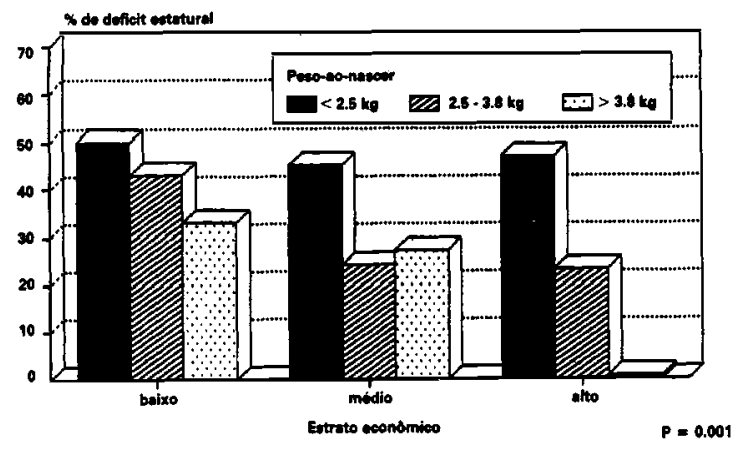

Fig. 3 - Associação de peso ao nascer e déficit estatural da criança, por estrato econômico, favela da Rocinha, Rio de Janeiro, RJ, 1986.

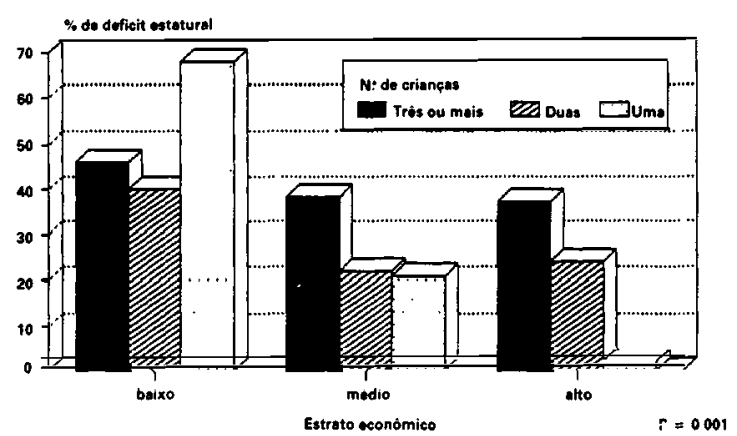

Fig. 4 - Associação de paridade materna e déficit estatural da criança, por estrato econômico, favela da Rocinha, Rio de Janeiro, RJ, 1986.

ocorrendo uma concentração de casos no estrato mais baixo.

O peso ao nascer também se mostrou um bom discriminante. Uma criança nascida com um peso inferior a $2,5 \mathrm{~kg}$ tem uma chance notavelmente maior de déficit de crescimento em relação àquela nascida com mais de $3,8 \mathrm{~kg}$. Em termos populacionais, isto significa que no primeiro estrato natiponderal, $30 \%$ excediam o esperado de $15,9 \%$ abaixo de -1DP, enquanto apenas $3 \%$ se encontravam no segmento das crianças com peso ao nascer acima de $3,8 \mathrm{~kg}$. Um perfil semelhante foi encontrado quando se controlou por estrato econômico (RPC-a* de 3,4 [I.C. $95 \%, 1,5-7,7$ ] entre as duas categorias extremas), sugerindo que o possível efeito do peso de nascimento ocorre independentemente das condiçôes gerais da familia (Figura 3 ).

A paridade também se mostrou associada com déficit estatural. Uma criança com pelo menos mais dois irmãos vivendo no mesmo domicílio tinha maior chance de crescimento retardado (Tabe-

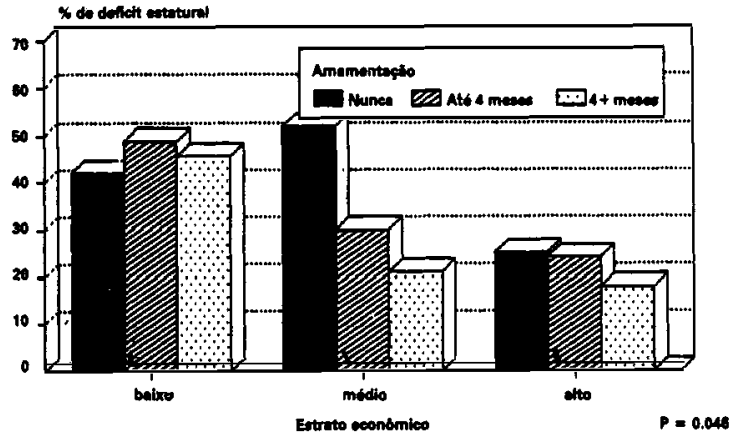

Fig. 5 - Associação de aleitamento materno e déficit es tatural da criança, por estrato econômico, favela da Rocinha, Rio de Janeiro, RJ, 1986.

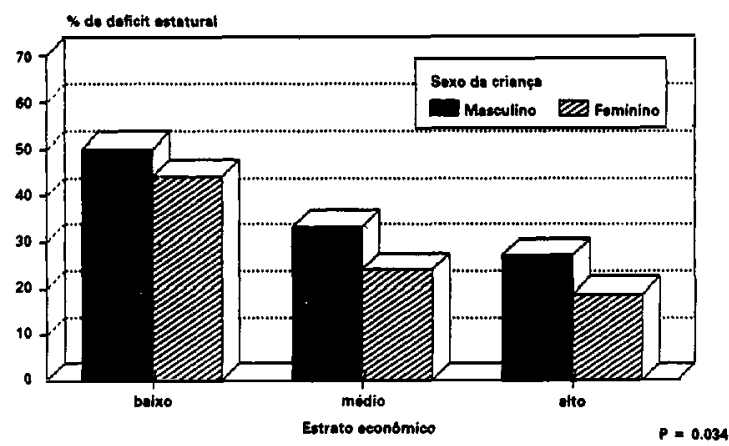

Fig. 6 - Associação de sexo e deficit estatural da criança, por estrato econômico, favela da Rocinha, Rio de JaneiIo, $\Gamma^{-} 1986$.

la 3). No entanto, este padrão não foi homogêneo entre os estratos econômicos como mostra a Figura 4. A tendência esperada - menor taxa entre os filhos (as) únicos (as) - ocorreu nos estratos médio e alto (respectivamente, RPC-a de 2,3 [I.C. 95\%, 0,4 14,2] e 25,4 [.C. $95 \%, 2,4-268,8]^{* *}$ à comparação das categorias de paridade $\geq 3$ v.s. 1). Inversamente, no estrato baixo, houve uma inflação de crianças únicas com retardo de crescimento (RPC-a de 0,4 [I.C.95\%, 0,2-2,9]). As demais variáveis - grau de escolarização materna, perda prévia de filhos, sexo da criança, intervalos interpartais e histórico de amamentação - também se mostraram associadas ao retardo de crescimento, ainda que de forma menos marcante do que as já referidas (Tabela 3 ). Ao controle por estrato econômico (Figuras 5 a 10) verificou-se um enfraquecimento das associações, à exceção de duas variáveis: histórico de amamentação, que evidenciou uma razão de produtos cruzados ajustada de 2,3 [I.C. 95\%, 1,1-4,4] entre as crianças que nunca amamentaram e as que aleitaram ao peito exclusivamente até pelo menos o fim do quarto mês de vida, e sexo da criança, com uma

\footnotetext{
* Razão de Produtos Cruzados ajustados.

* Os valores elevados e o largo intervalo de confiança encontrados são decorrentes de algumas celas com poucas observações, que causam uma considerável instabilidade aos estimadores.
} 


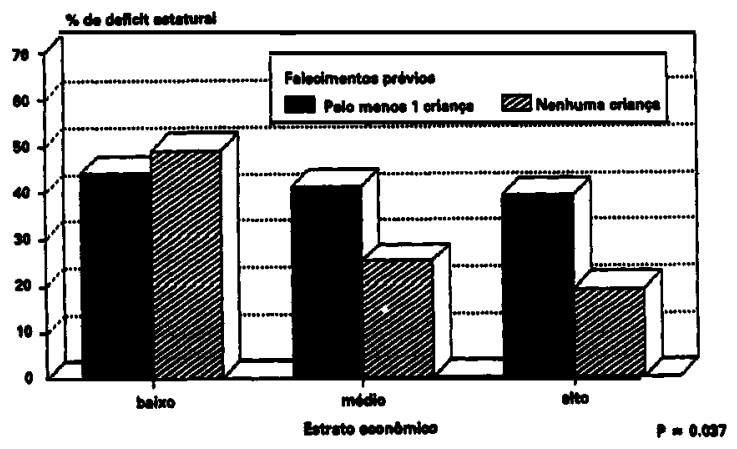

Fig. 7 - Associação de morte intantil prévia na famullia e défict estatural da criança, por estrato econômico, favela da Rocinha, Rio de Janeiro, RJ, 1986.

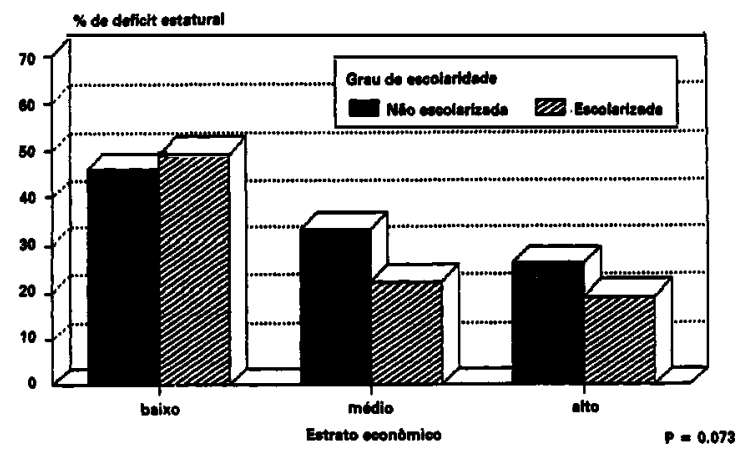

Fig. 8 - Associação de escolaridade materna e déficit estatural da criança, por estrato econômico, favela da Rocinha, Rio de Janeiro, RJ, 1986.

RPC-a de 1,5 [.C. 95\%, 1,1-2,1]. Do ponto de vista da significância estatística das associaçőes $\alpha=0,05$ năo houve alteração nesta última variável e na variável "perda prévia de filhos" (RPC-a de 1,6 [..C.5\%, 1,05-2,A]). Em contrapartida, após controle por estrato econômico, deixaram de ter significância estatística, assim sugerindo uma não rejeição da hipostese nula de homogeneidade (da năo diferença entre as estaturas das populações infantis nos estratos da variável testada), as associaçōes com escolaridade (RPC-a de 1,4 [1.C.95\%, 0,95-2,1]), intervalo interpartal subseqüente (RPC-a de 1,4 [I.C.95\%, 0,82,2]) e prévio (RPC-a de 1,5 [1.C.85\%, 0,95-2,2]).

Assim, para a comunidade em questão, podese tipificar uma criança sob risco de retardo de crescimento como sendo (a) oriunda de um domicillio de baixíssimo padrão econômico (ambiente físico); (b) preferencialmente um menino; (c) nascido com menos de $2,5 \mathrm{~kg}$; (d) que nunca tenha aleitado ao peito; (e) vivendo numa família com história de mortalidade infantil pregressa; e $(f)$ convivendo com pelo menos mais dois irmãos.

\section{DISCUSSÃO}

A julgar pelo parâmetro de peso-para-idade,

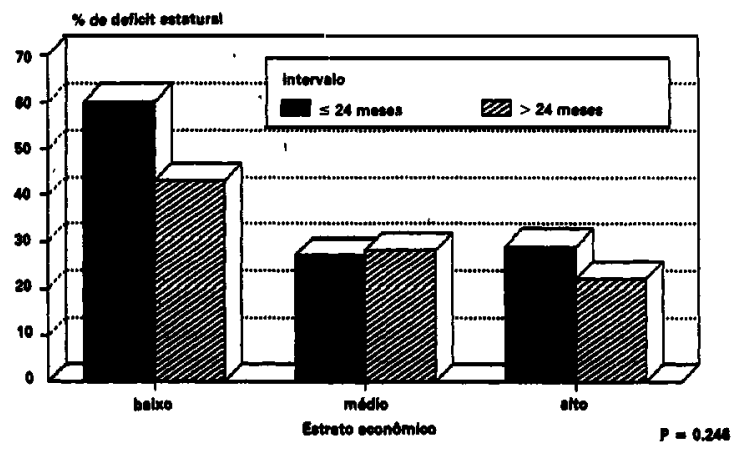

Fig. 9 - Associação de intervalo interpartal subseqüente e déficit estatural da criança, por estrato econômico, favela da Rocinha, kio de Janeiro, RJ, 1986.

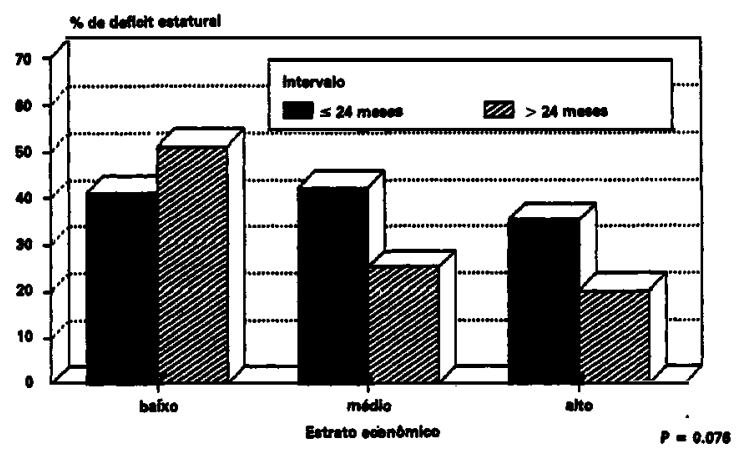

Fig. 10 - Associação de intervalo interpartal prévio e déficit estatural da criança, por estrato econômico, favela da Rocinha, Rio de Janeiro, RJ, 1986.

os dados expostos coincidem notavelmente com o achado de Monteiro e col. ${ }^{33}$, no município de São Paulo, onde $26 \%$ das crianças com menos de cinco anos encontravam-se abaixo do ponto de corte de $90 \%$ da mediana e nenhuma criança abaixo de $60 \%$ da mediana. $O$ perfil revelado na comunidade em estudo está de acordo com a noção de melhora do quadro nutricional geral na regiåo, contrastando marcamente com achados no nordeste do país, onde cerca de $70 \%$ tem alguma forma de desnutriç̄õo, sendo um terço de tipo moderado e severo ${ }^{32,41}$.

O perfil nutricional encontrado é compatível com um quadro geral de taxas de mortalidade infantil intermediárias como o do sudeste do país. Nesta situação também são menơres as agressర̃es da carência alimentar extrema e das infecçōes severas, permitindo que, neste novo patamar de problemas de moderada intensidade, as crianças se adaptem reduzindo o crescimento linear sem alterar a relação apropriada de peso-para-altura ${ }^{31}$. Assim, à medida que os níveis de desnutrição aguda declinam, os retardos estaturais passam a ser dominantes e de crescente preocupação. Na Rocinha, aproximadamente uma em cada três crianças encontrava-se abaixo do ponto de corte de -1DP, conquanto somente uma em seis é esperada. Em con- 
traste, havia apenas $2 \%$ mais crianças abaixo do ponto de corte de -2DP do que o esperado. A combinação destes achados mostra que a situação nutricional, em termos agregados, não chega a alarmar, mas que um impedimento nutricional sutil levando a uma deficiência crônica de crescimento é a tendência emergente. Entretanto, qualquer generalização para outras comunidades, principalmente às incipientes da periferia metropolitana, deve ser cautelosa. Possivelmente, o perfil encontrado na Rocinha seja paradigma de um tipo de comunidade grande e bem estabelecida. Estas questões carecem de maior aprofundamento.

Ainda que o problema verificado seja um de menor conspicuidade do que nas regiōes mais pobres do país e de outros países do Terceiro Mundo, 0 presente estudo ainda guarda uma clara determinação econômica ao fundo do déficit estatural verificado. Isto pode ser interpretado tanto sob a otica das condiçóes ambientais do domicilio na sua forma nominal, quanto do ponto de vista desta variável como um indicador mais amplo do "status" econômico-financeiro. Por exemplo, a renda domiciliar no estrato inferior (domicílios de baixo padrão ambiental) era de 0,49 salário-mínimo per capita, enquanto a de uma família no estrato superior (domicílios de bom padrão ambiental) era de 1,2 salário-mínimo per capita*. Considerandose que, à época, o poder aquisitivo mínimo de uma familia média de quatro indivíduos para a cesta básica, transporte e vestuário estava estimado $\mathrm{cm}$ dois salários mínimos ${ }^{46}$, deve-se concluir que pelo menos o quinto correspondente às famílias com más condiçбcs ambientais encontrava-se vivendo marginalmente ou abaixo da linha de pobreza especificada acima.

Dentro deste quadro de carência se situam alguns outros fenômenos. Tanto a alta paridade quanto o baixo peso ao nascer têm relação com a associação entre uma história de fracasso no alcitamento natural e um déficit de crescimento. Recémnascidos de baixo peso frcqüentemente apresentam dificuldades de amamentação. Esta é ainda mais prejudicada pela diminuida disponibilidade do tempo maternal quando existem múltiplos filhos para criar. Entretanto, como foi anteriormente apresentado, crianças únicas no estrato econômico inferior têm um elevado risco de retardo estatural.
Isto sugere que neste subgrupo, o efeito de competição entre irmãos pela mãe (supervisão) ou nutrientes não deve estar implicado. Possivelmente estas são as famílias onde a carência é extrema e, apesar da aparente limitação artificial do número de filhos, os recursos não se mostram sequer suficientes para promover uma alimentação adequada.

Existe uma tendência de as crianças nascidas de baixo peso serem de familias do mais baixo estrato econômico. Enquanto que $9,4 \%$ havia nascido de baixo peso no estrato alto, $12,5 \%$ havia nascido de baixo peso no estrato inferior**. Isto sugere que o baixo peso ao nascer tem uma atuação indireta pela sua preponderância no grupo economicamente desfavorecido e uma biológica direta como mostram os resultados da análise controlada. Deve se levar em conta que as crianças nascidas de baixo peso tendem a ser também aquelas que sofrem das desvantagens cconômicas após o nascimento.

Crianças do sexo masculino apareceram como vulneráveis ao desenvolvimento de déficit estatural. Outros estudos também têm mostrado uma associação com sexo. Enquanto alguns estudos da África e Ásia mostraram uma proteçăo para o sexo masculino $0^{36,45}$, o oposto foi encontrado no ocidente ${ }^{10}$, 43. Ainda que a falta de associação seja o mais comum nos estudos da América Latina ${ }^{29}$, o presente achado está mais de acordo com o estudo descrito por Romafra e col. no Peru ${ }^{43}$. Os eventos subjacentes a estes resultados contraditórios são de difícil explicação e merecem mais investigaçăo.

O determinismo econômico sobre o déficit estatural tem sido descrito na literatura $36,46,52$. Ainda que outros estudos intracomunitários tenham mostrado uma rclação inversa entre a desnutrição e o nível econômico ${ }^{13}, 40,43,48,53$ este fenômeno tem. sido pouco documentado no Brasil, onde se avolumam as comunidades de baixa renda de grande magnitude. A medida que estas crescem em dimensōcs e se tornam bem estabelecidas, elas tendem também a se diferenciar internamente do ponto de vista econômico. Ao replicarem o perfil da sociedade como um todo, grandes aglomerados de baixa renda devem, pois, ser encarados como cidades dentro de cidades. Segue daí a noção de

* Utilizando o método de análise de variança (ANOVA), csta diferença se mostra significante a p=0.0001.

* Este achado deriva de uma análise primária dos dados. Apesar de esta diferença não ser estatisticamente significante em funçăo do tamanho amostral $(\mathrm{p}=0.245)$, outros estudos têm verificado diferenças de baixo pesoao-nascer entre estratos sociais da mesma ordem (ver referências 21 no final deste texto, [Barros FC, Victora CG, Granzoto JA et al. Saúde perinatal em Pelotas, RS: fatores sociais e biológicos. Revista de Saúde Pública, S. Paulo 1984; 18:301-312], [Binsacca DB, Ellis J, Martin DG, Petitti DB. Factors associated with low birth weight in an inner-city population: the role of financial problems. American Journal of Public Health, 1987; 77:505-506] e [Lieberman E, Ryan KJ, Monson RR, Shoenbaum SC. Risk factors accounting for social differences in the rate of premature birth. The News England Journal of Medicine, 1987; 317:743-748]. Ainda que não se possa acatar o resultado com a segurança desejada, sua aceitação respalda-se em última análise da consistência externa do achado. 
"favela dentro de favela". Na Rocinha, nada menos que 12.000 individuos encontram-se em clara desvantagem. Como mostra o presente estudo, este desnivelamento tem implicaçōes importantes no perfil intracomunitário da deficiência de crescimento. Conseqüentemente, a questão da priorização de açőes de prevenção e a alocação adequada de recursos para grupos vulneráveis se faz tanto necessária no planejamento do setor saúde na sociedade em geral, como em comunidades tais quais a aqui estudada.

Finalmente, cabe ressaltar que avaliaçóes comunitárias como a do tipo descrita aqui são da maior pertinência na consolidação de propostas locais de intervenção e que, certamente, terão um papel de destaque no contexto da descentralização do setor saúde ora em curso no Brasil ${ }^{12}$, à implantação dos distritos sanitários ${ }^{47}$.

REICHENHEIM, M.E. \& HARPHAM, T. [An intra-community profile of nutritional deficiency: a study of under-fives in a low-income community in Rio de Janeiro (Brazil)], Rev. Saúde públ. S. Paulo, 24: 69-79, 1990.

ABSTRACT: This study is part of a larger epidemiological study concerned with the health status of children under the age of five carried out in the squatter settlement of Rocinha, and focuses on the nutritional profile of a representative sample of 591 children. According to the weight-for-age criteria (Gomez's classification), $23,9 \%$ and $2,0 \%$ were, respectively, mildly and moderately malnourished. This finding is in agreement with the assessment using weight-for-height and height-for-age as anthropometric indicators: (a) absence of acute malnutrition (wasting) indicated by a pattern overlapping that of an expected normal population, and (b) growth deficiency (stunting) indicated by $7 \%$ and $15 \%$ of children exceeding the proportion normally expected to be, respectively, below the -1 and -2 standard deviate limits. So far as growth failure was concerned, the following variables remained associated even when controlling for economic'status (indicated by the environmental conditions of the household): low birth weight, number of siblings equal to or above three, male gender, a history of never having breastfed and a family history of previous sibling death. Each variable is discussed separately, as well as the overall nutritional profile and the marked social intra-community stratification related to growth deficit.

KEYWORDS: Nutritional status. Body height. Body weight. Growth.

\section{REFERÊNCIAS BIBLIOGRÁFICAS}

1. ARMITAGE, P \& BERRY, G. Slatistical methods in medical research. $2^{\text {nd }}$ ed. Oxford, Blackwell Scientific Publications, 1987.

2. AUSTDN, J.E. Confronting urban malnutrition: the design of malnutrition programmes. Baltimore, John Hopkins University Press, 1980. (World Bank Staff Occasional Papers, N2 28).

3. BASTA, S.S. Nutrition and health in low income urban areas of the third world. Ecol. Food Nutr., 6: 113-24, 1977.

4. BATISTA-FILHO, M.; LUCENA, M.A.F.; COELHO, H. A.L. Desnutrição protéico-energética em três cidades brasileiras: São Luís, Recife e São Paulo. Bol. Ofic. sanit. panamer., 90: 48-57, 1981.

5. BOGIN, B.A. \& MACVEAN, R.B. Growth in height and weight of urban Guatemalan primary school children of low and high socio-economic class. Hum.Biol., 50: 477-87, 1978.

6. BREILH, J.; GRANDA, E.; CAMPAÑA, A.; BITENCOURT, $O$. Clase social y desigualdad ante la muerte en Quito. In: Breilh, J.; Granda, E.; Campaña, A.; Betancourt, O., eds. Ciudad y muerte infantil. Quito. CEAS Ediciones, 1983. p. 83-126

7. BRINK, E.W.; ALY, H.E.S.; DAKROURY, A.M; SAID, A.K.; GHONEME, F.M.; HUSSEIN, M.A.; SHAHEEN, F.M.; PECK, R.E.; GENTRY, E.D.; NI CHA-
MAN, M.Z. The Egyptian National Nutrition Survey, 1978. Bull. Wld Hlih Org., 61: 853-60, 1983.

8. CAVALLIERI, P.F. Favelas cariocas: mudança na infraestrutura. Rio de Janciro, Instituto de Plancjamento Municipal - IPLANRIO, 1986.

9. CENTER FOR DISEASE CONTROL (CDC). Anthropometric softwere package: system documentation. Atlanta, Ga., sd.

10. CHINN, S. \& RONNA, R.J. The secular trend in the height of primary school children in England and Scotland from 1972 to 1980. Ann. Ilum. Biol., 11: 1-16, 1984.

11. CLARK, W.F. The rural to urban nutritional gradient: application and interpretation in a developing nation and urban situation. Soc. Sci. Med., 14D: 31-6, 1980.

12. CORDEIRO, H. A reforma sanitária: das ações integradas de saúde aos sistemas unificados e descentralizados de saúde. Cad. IMS, Rio de Janeiro, 2: 9-14, 1988.

13. CREAN, J.T.G.; LING, S.C.; PEARSON, D.J.; SHAW, S.N.; STEWART, R.J.; WILSON, J.H. Dhavari Project 1985: an investigation into patterns of infant feeding and malnutrition in the urban slum of Dhavari in Bombay. J. trop. Pediat., 33 (suppl. 2) 1987.

14. DESAI, I.D.; GARCIA TAVARES, M.L.; DUTRA DE OLIVEIRA , B. S.; DOUGLAS, A.; DUARTE, 
F.A.M.; DUTRA DE OLIVEIRA, J.E. Food habits and nutritional status of agricultural migrant workers in Southem Brazil. Amer. J. clin. Nutr., 33: 702-14, 1980.

15. DOREA, J.G.; ALMEIDA, I.S.; QUEIROZ, E.F.O.; HORNER, M.R. Nutritional status and zinc nutriture in infants and children in a poor urban community of Brazil. Ecol. Food Nutr., 12: 1-6, 1982.

16. FRANKLN, R.R.; BERTRAND, W.E.; NKAMANY, K. A cross sectional study of wasting and stunting in Kinshasa, Zaire. Ann. Soc. belg. Med. trop., 64: 403-11, 1984.

17. GOMEZ, F.; GALVAN, R.R.; FRENK, S.; CRAVIOTO, J.; CHÁVES, R.; VAZQUEZ, J. Mortality in second and third degree malnutrition. J. trop. Pediat., 2: 77-83, 1956.

18. GRAHAM, G.G.; MACLEAN Ir., W.C.; KALLMAN, C.H.; RABOLD, J.; MELLITS, E.D. Urban-rural differences in the growth of Peruvian children. Amer. J. clin. Nutr., 33: 338-44, 1980.

19. GRAITCER, P.L. \& GENTRY, M. Measuring children: one reference for all. Lancet, 2: 297-9, 1981.

20. GRIMBLE, R.F. A study of the pattem of clinical proteinenergy malnutrition in Accra from 1970 to 1978. J. trop. Pediat., 27: 12-4, 1981.

21. HABICHT, J.-P.; MARTORELL, R.; YARBROUGH, C.; MALINA, R.M.; KLEIN, R.E. Height and weight standards for pre-school children. How relevant are ethnic differences in growth potential. Lancet, 1: 611-5, 1974.

22. HARPHAM, T.; LUSTY, T.; VAUGHAN, P. In the shadow of the city: community health and the urban poor. Oxford, Oxiord University Press, 1988.

23. JANSEN, G.R.: JANSEN, N.B.; SHIGETOMI, C.T.; HARPER, J.M. Effect of low income and geographic region on the nutritional value of diets in Brazil. Amer. J. clin. Nutr., 30: 955-64, 1977.

24. KOOPMAN, F.S.; FAGARDO, L.; BERTRAND, W. Food, sanitation and the socio-economic determinants of child growth in Colombia. Amer. J. publ. Hlth, 71: 31 . $7,1981$.

25. KRUMDIEK, C.L. The rural to urban malnutrition gradient. J. Amer. med. Ass., 215: 1652-4, 1971.

26. LIBERATOS, P.; LINK, B.G.; KELSEY, J.L. The measurements of social class in epidemiology. Epidem. Rev., 10: 87-121, 1988.

27. LUSTOSA, T.Q. Avaliação antropométrica. In: Fundação IBGE. Perfil estatístico de crianças e mäes no Brasili:aspectos nutricionais, 1974-1975. Rio de Janeiro, 1982. p. 83-148.

28. MARQUES. R.M.; BERQU6, E.; YUNES, J.; MARCONDES, E. Crescimento de crianças brasileiras: peso e altura segundo idade e sexo. Influência de fatores sócioeconômicos. An. Nestlé, (84, supl. 2), 1974.

29. MARTORELL, R. \& HABICHT, J.-P. Growth in early childhood in developing countries. In: Falkner, F. \& Tanner, J.M. eds. Human growth - a comprehensive treatise. Vol. 3: Methodology, ecological, genetic, and nutritional effects on growth. New York, Plennum Press, 1986. p. 241-62.

30. MARTORELL, R.; KENDALL, C.; FOOTE, D.R. Growth and poverty in Honduras. [Paper presented at the Fifty-second Annual Meeting of the American Association of Physical Anthropologists, Indianapolis, Indiana, 1983].

31. MARTORELL, R.; LESLIE, J.; MOOCK, P.R. Characteristics and determinants of child nutritional status in $\mathrm{Ne}$ pal. Amer. J. clin. Nutr., 9: 74-86, 1984.

32. MONTEIRO, C.A.; BENICIO, M.H.D'A. Epidemiologia da desnutrição protéico-calórica. In: Nóbrega, F.J., ed. Desnutriçāo intra-uterina e pós-natal. Rio de Janeiro, Panamed Editora, 1981. p. 110-30.

33. MONTEIRO, C.A.; BENICIO, M.H.D'A.; ZUNIGA, H.P.P.; SZARFARC, S.C. Estudo das condiçōes de saúde das criancas do Município de São Paulo, SP (Brasil), 1984-1985. II - Antropometria nutricional. Rev. Saúde públ., S. Paulo, 20: 446-53, 1986.

34. MORELL, S. \& MORELL, D. Six slums in Bangkok: problems of life and options for actions. Bangkok, UNICEF, 1972.

35. MORLEY, D.C. \& WOODLAND. M. See how they grow: monitoring child growth for appropriate health care in developing countries. London, Macmillan Press, 1983.

36. NABARRO, D.; HOWARD, P.; CASSEL, C.; PANT, M.; WLGA, A; PADFIELD, N. The importance of infections and environmental factors as possible determinants of growth retardation in children. In: Waterlow, J.C., ed. Linear growth retardation in less developed countries. New York, Vevey/Raven Press, 1988. p.16585. Nestlé Nutrition Workshop Series, v. 14).

37. NNANYELUGO, D.O. Urbanization and nutrition. Afr. Hith, 2: 15-6, 1980

38. POWELL, C.A.; GRANTHAM-MACGR.EGOR, S. The ecology of nutritional status and development in Kingston, Jamaica. Amer. J. clin. Nutr., 4: 1322-31, 1985.

39. PRASADA-RAO, T.M.; GOWRINATH-SASTRY, J.; VIJAYARGHANSAN, K. Nutritional status of children in urban slums around Hyderabad City. Indian J. med. Res., 62: 1492-9, 1974

40. RAMOS-JIMENEZ, P.; CHIONG-JAVIER, M.E.; SEVILLA, J.C. Health and nutritional problems and the utilisation of health services. Manila, 1986. (RC-DLSU Research Report № 2, v. I).

41. REICHENHEIM, M.E. Young children's nutrition in an urban, low-income community of Northeast Brazil: what do mothers do, health agents know, and pediatricians advise. London, 1984. [Tese de Mestrado (M.Sc.) - Institute of Child Health. University of London].

42. REICHENHEIM, M.E. Child health in an urban context: riskfactors in a squatter settlement of Rio de Janeiro. 
London, 1988. [Tese de Doutorado (Ph.D.) - Institute of Child Health \& London School of Hygiene and Tropical Medicine,University of London].

43. ROMANA. G.L.; BROWN, K.H.; BLACK, R.E.; KANASHIRO, H.C. Longitudinal studies of infectious diseases and physical growth of infants in Huascar, and underprevileged periurban community in Lima, Peru. Amer. J. Epidem., 129: 769-84, 1989.

44. ROSSI-ESPAGNET, A. Primary health care in urban areas: reaching the urban poor in developing countries; A State of the Art Report by UNICEF and WHO $n^{\circ} 2499 \mathrm{M}$. Geneva, World Health Organization, 1984.

45. SABIR, N.I. Why do girls die more? Sex differences in growth and child-rearing practices in a slum area of Lahore. London, 1984. [Tese de Mestrado (M.Sc.) - Institute of Child Health. University of London].

46. SHRIMPTON, R. Ecologia da desnutriçâo no infância: análise da evidência das relaçôes entre variáve is sócio. econômicas e estado nutricional. Brasília, CNRH/IPEAUNICEF, 1986. (Série Instrumentos para $A c ̧ a ̃ o ~ n^{\circ} 3$ ).

47. SILVEIRA, T.R.; LEMME, A.C.; FAGUNDES, C.G.; SILVA, C.L.S.; ROSSI, D.R.; NASCIMENTO, D.R.; CARDOSO, F.; ALMEIDA, H.G.; XAVIER, I.M.; RIBEIRO, J.C.; LIMA, K.M.L., TROTTA, L.; FERREIRA, L.H.M., ZIBECCHI, E.; FREIRE, P.; OLIVEIRA, R.M.; LEIBOVICI, Z. Distrito sanitário: contribuição para um novo tipo de atendimento dos serviços de saúde. Cad. IMS, Rio de Janeiro, 2: 114-50, 1988.

48. SINGHAL, P.K.; MATHUR, G.P.; MATHUR, S.; SINGH, Y.D. Mortality pattems in under six children in ICDS urban slum. Indian Pediat., 23: 617-22, 1986.

49. STATISTICAL ANALYSIS SYSTEM (SAS). User guide. Cary, NC, SAS Institute, 1985.

50. SUDHARTO, P. Experiences in the field of urban primary health care in Jakarta; Report of the joint UNICEF/ WHO meeting on primary health care in urban areas. Geneva, 1983. (WHO Document SHS/HSR/83.1).

51. SYSTEM FOR INFORMATION RETRIEVAL/DATA BASE MANAGEMENT SYSTEM (SIR/DBMS). User manual, version 2, 1980.

52. TANNER, J.M. Foetus into man. London, Open Books, 1978.
53. TEKCE, B. \& SHORTER, F. Socio-economic determinants of child mortality and intermediary processes: findings from a study of squatter settlements in Amman. Pop. Develop. Rev., 10 (Suppl.): 257-80, 1984.

54. UNDERWOOD, B.A. \& HOFVANDER, Y. Appropriate timing for complementary feeding of the breast-fed infant: a review. Acta paediat. scand., 294 (Suppl.): 5-32, 1982.

55. VALVERDE, V.; NIEVES, I.; SLOAN, N.; PILLET, B.; TROWBRIDGE, F.; FARRELL, T.; BEGHIN, I.; KLEIN, R.E. Life styles and nutritional status of children from different ecological areas of El Salvador. Ecol. Food Nutr., 9: 167.77, 1980.

56. VICTORA, C.G.; BARROS, F.C.; VAUGHAN, J.P.; MARTINES, J.C.; BERIA, J.U. Birthweight, socioeconomic status and growth of Brazilian infants. Ann. hum. Biol., 14: 49-57, 1987.

57. WATERLOW, J.C. \& THOMPSON, A.M. Observations on the adequacy of breast-feeding. Lancet, 2:238-41, 1979.

58. WATERLOW, J.C.; ASHWORTH, A.; GRIFFITHS, M.F. Faltering in infant growth in developing countries. Lancet, 2: 1176-8, 1980.

59. WOLFE, B.L. \& BEHRMAN, J.R. Determínants of child mortality, health and nutrition in a developing country. J. Develop. Econ., 11: 163-93, 1982.

60. WORLD HEALTH ORGANIZATION. Measuring change in nutritional status: guidelines for assessing the nutritional impact of supplmentary feeding programmes for vulnerable groups. Geneva, 1983.

61. WORLD HEALTH ORGANIZATION. Working Group. Use and interpretation of anthropometric indicators of nutritional status. Bull. Wld Hlth Org.; 64: 929-41, 1986.

62. WRAY, J.D. Nutrition and health in urban slums: an over view. [Paper prepared for a Workshop on Community Health and the Urban Poor, org. by the London School of Hygiene and Tropical Medicine, OXFAM, and UNICEF, 7-12 July 1985, Oxford, Engl., 1985].

Recebido para publicação em 1/8/1989 Aprovado para publicação em $7 / 11 / 1989$ 